Die Kolumne $m_{\mathscr{d}_{r}}$ gibt die auf $A=$ I reduzierten Gesamthelligkeiten des Kometen. Nach einer graphischen Ausgleichung hat $m_{r}$ während der Beobachtungszeit von $6^{\mathrm{m}} \cdot 9$ bis $5^{\mathrm{m}} \circ$ zugenommen. Da $\log r$ in derselben Zeit nur um 0. I $5^{6}$ abgenommen hat, scheint die Helligkeit des Kometen sich also mit der fünften Potenz der Distanz von der Sonne zu verändern.

Treptow-Sternwarte, 19 1 I Sept. 2 2. F.S. Archenhold.

Die folgenden Helligkeitsschätzungen des Kometen ig I c (Brooks) habe ich mit einem Zeißschen Prismenbinokel mit 8 facher Vergrößerung in Alupka (Krim) nach der Argelanderschen Methode angestellt. Als Vergleichsterne benutzte ich einige BD-Sterne.

\begin{tabular}{|c|c|c|}
\hline $\begin{array}{c}\text { 19II } \\
\text { Aug. I } 9\end{array}$ & $\begin{array}{l}\text { M. Z. Simeis } \\
10^{\mathrm{h}} 16^{\mathrm{m}}\end{array}$ & $\begin{array}{r}\mathrm{H} \\
6^{\mathrm{m}} \cdot 7\end{array}$ \\
\hline 20 & 55 & $6.3 \mathrm{I}$ \\
\hline $2 \mathrm{I}$ & $4 \mathrm{I}$ & 6.15 \\
\hline 24 & 933 & 5.75 \\
\hline 25 & $\begin{array}{l}9 \\
5\end{array}$ & 5.70 \\
\hline
\end{tabular}

Die Beobachtungen, welche fortgesetzt werden, sollen später ausführlich mitgeteilt werden.

Simferopol, 19 I S Sept. 25.

L. Filippoff.

De las observaciones visuales y fotográficas efectuadas en el Observatorio de Madrid resulta que el cometa de Brooks ha ido aumentando constantemente de tamaño y de brillo desde su aparición. A fin de Septiembre se observa á simple vista como una estrella de magnitud comprendida entre la tercera y la segunda.

El núcleo con la nebulosidad que lo circunda ha llegado á un diámetro de $2 \mathrm{I}^{\prime}$ y la cola se extiende hasta unos $\mathrm{I} 5^{\circ}$.

La cola está formada por filamentos muy finos entrelazados, formando un ángulo muy agudo; por uno $\mathrm{y}$ otro lado de ella emite el-núcleo otros filamentos más cortos, habiendo llegado como maximum á una distancia de $3^{\circ}$ del núcleo: es de constitución bastante uniforme.

El examen del espectro por medio de un espectroscopio de rendija permite apreciar tres imágenes, en el amarillo, en el verde y en azul, siendo la verde la más intensa. Las fotografras del mismo espectro obtenidas con prisma objetivo en la ecuatorial fotográfica de Grubb, empleando placas isocromáticas, reproducen además de las tres imágenes visibles otras cuatro, dos de las cuales estan formadas por distintas rayas espectrales, hallándose formada la cuarta imágen por tres rayas y la sexta por cinco: la séptima imagen parece doble, pero no se definen netamente las componentes.

El espectro de la cola no es visible y en las placas se insinúa débilmente en las tres primeras imágenes.

Longitudes de onda, intensidad y disposición de las rayas espectrales, como resultan de las medidas efectuadas sobre cuatro placas obtenidas en los dias 19,25 y 26 de septiembre:

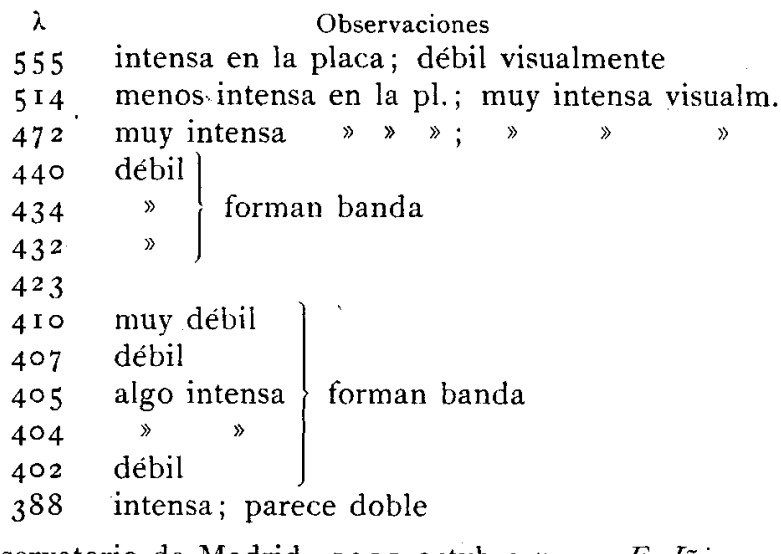

Observatorio de Madrid, I 9 I I octubre 7 . F. Iñguez.

\title{
Efemeride della cometa 1911 c (Brooks).
}

Cogli elementi parabolici che trovansi in A. N. 4536 ho calcolato la seguente efemeride approssimata della cometa. É probabile che lo splendore reale sia minore di quello corrispondente alle grandezze indicate.

I $2^{\text {h }}$ Berlino.

\begin{tabular}{|c|c|c|c|c|c|c|}
\hline \multicolumn{2}{|c|}{ I 912} & $\alpha$ vera & $\delta$ vera & $\log r$ & $\log d$ & Gr. \\
\hline \multirow[t]{12}{*}{ Gen. } & 7 & $\mathrm{I} 4^{\mathrm{h}} 47^{\mathrm{m}}$ I $5^{\mathrm{s}}$ & $-45^{\circ} 32^{\prime} \cdot 7$ & \multirow[t]{4}{*}{$0.1922 \mathrm{I}$} & \multirow[t]{4}{*}{$0.266 \mathrm{IO}$} & \multirow[t]{4}{*}{$8^{\mathrm{m}} \cdot \mathrm{I}$} \\
\hline & 8 & $48 \quad 44$ & $45 \quad 52.0$ & & & \\
\hline & 9 & $50 \quad 12$ & 46 I I.I & & & \\
\hline & 10 & 5 I 38 & $46 \quad 30.0$ & & & \\
\hline & I I & 5.32 & $\begin{array}{ll}46 & 48.7\end{array}$ & \multirow[t]{4}{*}{0.20976} & \multirow[t]{4}{*}{0.27253} & \multirow[t]{4}{*}{8.2} \\
\hline & I 2 & $54 \quad 24$ & $47 \quad 7.2$ & & & \\
\hline & I 3 & 5544 & $47 \quad 25.5$ & & & \\
\hline & I 4 & 573 & $47 \quad 43.6$ & & & \\
\hline & I 5 & $58 \quad 19$ & $48 \quad$ I. 6 & \multirow[t]{4}{*}{0.22646} & \multirow[t]{4}{*}{0.27852} & \multirow[t]{4}{*}{$8 \cdot 3$} \\
\hline & I 6 & I $4 \quad 5935$ & 48 I 9.4 & & & \\
\hline & I 7 & I $5 \bigcirc 48$ & $4^{8} \quad 36.9$ & & & \\
\hline & I 8 & I 5 I $5^{8}$ & $-48 \quad 54 \cdot 3$ & & & \\
\hline
\end{tabular}

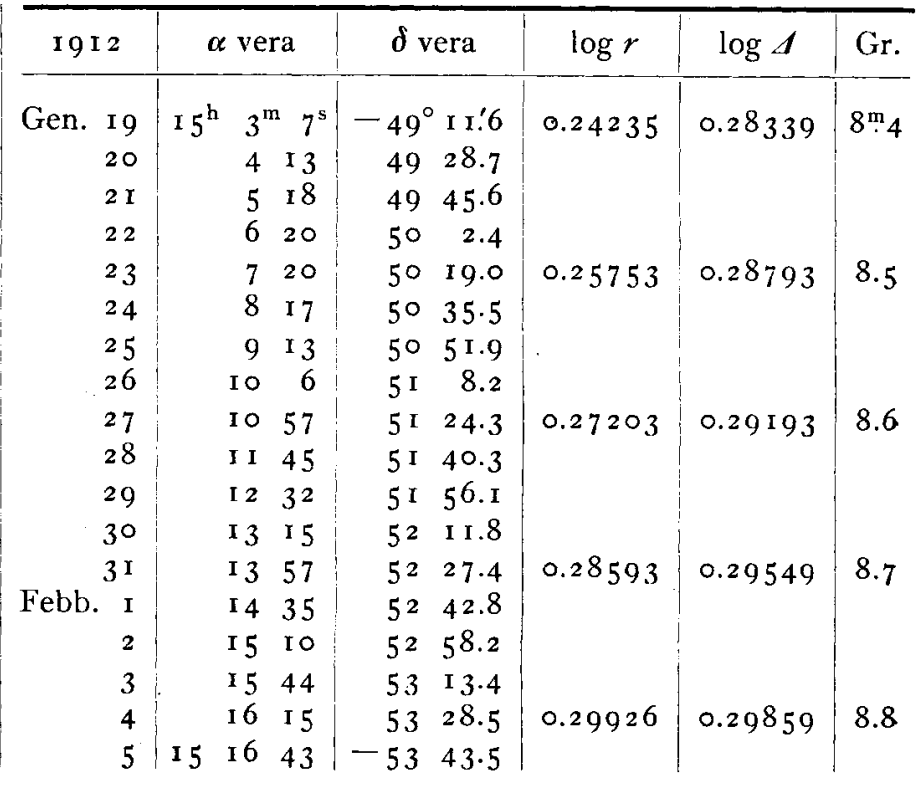




\begin{tabular}{|c|c|c|c|c|c|}
\hline I 9 I 2 & $\alpha$ vera & $\delta$ vera & $\log r$ & $\log \lambda$ & Gr. \\
\hline Febb. 6 & $\mathrm{I} 5^{\mathrm{h}} 17^{\mathrm{m}} 8^{\mathrm{s}}$ & $-53^{\circ} 5^{8} \cdot 3$ & \multirow{6}{*}{0.31206} & \multirow{6}{*}{$0.3013^{6}$} & \multirow{6}{*}{$8^{m} \cdot 9$} \\
\hline 7 & 1731 & $54 \times 3.0$ & & & \\
\hline 8 & 1750 & $54 \quad 27.6$ & & & \\
\hline 9 & I $8 \quad 7$ & $5442 . I$ & & & \\
\hline IO & I 82 I & $54 \quad 5^{6.4}$ & & & \\
\hline I I & I 83 I & $55 \quad 10.6$ & & & \\
\hline I 2 & I $8 \quad 39$ & $55 \quad 24.6$ & \multirow[t]{2}{*}{0.32437} & \multirow[t]{4}{*}{0.30378} & \multirow[t]{4}{*}{9.0} \\
\hline 13 & I 843 & $\begin{array}{ll}55 & 38.5\end{array}$ & & & \\
\hline I 4 & I 845 & $\begin{array}{lll}55 & 52.3\end{array}$ & \multirow{5}{*}{0.33622} & & \\
\hline I 5 & 1843 & $5^{6} \quad 6.0$ & & & \\
\hline 16 & $183^{8}$ & $5^{6} \quad \mathrm{r} 9.5$ & & \multirow[t]{4}{*}{0.30598} & \multirow[t]{3}{*}{9.0} \\
\hline I 7 & I $8 \quad 29$ & $5^{6} \quad 3^{2.9}$ & & & \\
\hline I 8 & I $8 \quad 17$ & $5^{6} 4^{6.1}$ & & & \\
\hline 19 & I $8 \quad 2$ & $5^{6} \quad 59.2$ & \multirow{4}{*}{$0.347^{67}$} & & \multirow{5}{*}{9.1} \\
\hline 20 & I 743 & $57 \times 2 . x$ & & \multirow[t]{3}{*}{0.30797} & \\
\hline 21 & $172 \mathrm{I}$ & $\begin{array}{lll}57 & 24.8\end{array}$ & & & \\
\hline 22 & 1655 & $57 \quad 37 \cdot 3$ & & & \\
\hline 23 & 1625 & $57 \quad 49.6$ & \multirow{3}{*}{$0.3587 \mathrm{I}$} & \multirow{3}{*}{0.30987} & \\
\hline 24 & I5 53 & $\begin{array}{ll}58 & 1.7\end{array}$ & & & \multirow[t]{2}{*}{$9 . I$} \\
\hline 25 & I 5 I $5 \quad 16$ & $-5^{8} \quad$ I 3.6 & & & \\
\hline
\end{tabular}

R. Osservatorio al Collegio Romano, 19 I I Dic. 7 .

\begin{tabular}{|c|c|c|c|c|c|}
\hline I 9 I 2 & $\alpha$ vera & $\delta$ vera & $\log r$ & $\log A$ & Gr. \\
\hline Febb.26 & $15^{\mathrm{h}} 14^{\mathrm{m}} 3^{6^{\mathrm{s}}}$ & $-58^{\circ}=5 \cdot 3$ & & & \\
\hline 27 & I 352 & $5^{8} \quad 36.8$ & & & \\
\hline 28 & I 34 & 5848.1 & 0.369 .39 & 0.31167 & $9^{m} \cdot 2$ \\
\hline 29 & I 2 I 3 & $5^{8} \quad 59.2$ & & & \\
\hline Marz. I & I I I 18 & 59 ro.o & & & \\
\hline 2 & 10 19 & $59 \quad 20.6$ & & & \\
\hline 3 & 15996 & $-59 \quad 30.9$ & $0.3797 \mathrm{r}$ & 0.31349 & $9 \cdot 3$ \\
\hline
\end{tabular}

L' astro fu stimato da Dr. G. Abetti di $6^{\mathrm{m}} \cdot 5$ il giono I dicembre IgI I.

Per un maggiore accordo del calcolo col cielo si possono applicare alle posizioni date dall'efemeride le correzioni empiriche seguenti:

$$
\begin{aligned}
& \lambda \alpha=+{ }_{1} 6^{\mathrm{s}}+0.5\left(t-t_{0}\right) \boldsymbol{A}_{0} / \boldsymbol{A}_{1} \cdot \sec \delta_{1} / \sec \delta_{0} \\
& \Lambda \delta=+2.4+0.06\left(t-t_{0}\right) \boldsymbol{A}_{0} / \boldsymbol{A}_{1}
\end{aligned}
$$

$A_{0}$, sec $\delta_{0}$ corrispondono a $t_{0}=$ I Dicembre I9I I

$$
\log A_{0}=0 . \mathrm{I} 6 \mathrm{I} \quad \log \sec \delta_{0}=0.060 .
$$

Verso il 3 marzo la correzione dovrebbe essere circa $+\mathbf{r}^{\mathrm{m}}+6^{\prime}$.

E. Millosevich.

\section{Eléments provisoires et éphéméride de la comète 1911 h (Schaumasse)}

par $G$. Fayet et $A$. Schaumasse.

Les observations utilisées sont celles obtenues à Nice les Nov. 30 (M. Schaumasse), Déc. I (M. M. Favelle et Schaumasse) et celle faite, le Déc. 2, à Arcetri.

Voici les éléments conclus:

$$
\begin{aligned}
& T=\text { I9 I } 2 \text { Février } 5.347 \text { t. m. Paris } \\
& \omega=109^{\circ} 7.6 \\
& \delta=\text { II } 5 \text { I } 2.1 \text { I } 911.0 \\
& i=2029.4 \\
& \log q=0.06822
\end{aligned}
$$

\begin{tabular}{|c|c|c|c|c|c|c|c|c|}
\hline \multicolumn{2}{|c|}{$191 I^{-12}$} & \multicolumn{2}{|r|}{$\alpha$} & \multicolumn{2}{|c|}{$\delta$} & $\log r$ & $\log \lambda$ & $\mathrm{I}: r^{2} \alpha^{2}$ \\
\hline Déc. & 28 & I $5^{h}$ & $\mathrm{o}^{\mathrm{m}} 25^{\mathrm{s}}$ & $-0^{\circ}$ & $50^{\prime}$ & 0.1207 & 0.2080 & 0.22 \\
\hline & 30 & & 855 & & 19 & & & \\
\hline Janv. & $\mathrm{I}$ & & 1730 & I & 49 & o. I I I 6 & 0.2008 & 0.24 \\
\hline & 5 & & $345^{r}$ & & 47 & & & \\
\hline & 9 & I 5 & $52 \quad 22$ & 3 & 43 & 0.0953 & o. 1899 & 0.27 \\
\hline & I 3 & I 6 & 102 & 4 & 37 & & & \\
\hline & 17 & & 2744 & 5 & 28 & 0.0822 & 0.1835 & 0.30 \\
\hline & $2 \mathrm{I}$ & I 6 & $45 \quad 24$ & & I 7 & & & \\
\hline & 25 & I 7 & 258 & 7 & 2 & $0.073^{2}$ & o. 18 I 3 & $0.3 \mathrm{I}$ \\
\hline & 29 & & 2023 & 7 & 43 & & & \\
\hline Févr. & 2 & & $373^{1}$ & 8 & $2 \mathrm{I}$ & 0.0689 & 0.1830 & $0.3 \mathrm{I}$ \\
\hline & 6 & $\mathfrak{I} 7$ & $54 \quad 23$ & 8 & 55 & & & \\
\hline & IO & I 8 & I0 53 & 9 & 25 & 0.0696 & 0.1877 & 0.30 \\
\hline & I 4 & & $26 \quad 59$ & 9 & $5 \mathrm{I}$ & & & \\
\hline & I 8 & & $42 \quad 39$ & 10 & I 4 & 0.0754 & O.I944 & 0.29 \\
\hline & 22 & I 8 & $57 \quad 53$ & 10 & 33 & & & \\
\hline & 26 & 19 & 1236 & Io & 48 & $0.085^{8}$ & 0.2023 & 0.27 \\
\hline Mars & $\mathbf{I}$ & & 2650 & II & I & & & \\
\hline & 5 & & $40 \quad 30$ & I I & I I & 0.0999 & 0.2107 & 0.25 \\
\hline & 9 & 19 & 5342 & I I & 19 & & & \\
\hline & I 3 & 20 & $6 \quad 22$ & I I & 25 & $0.117 \mathrm{I}$ & 0.2189 & $0.2 \mathrm{I}$ \\
\hline & I 7 & & I 830 & I I & 29 & & & \\
\hline & 21 & & 309 & I I & $3^{2}$ & o. 1 $3^{6} 3$ & 0.2265 & 0.19 \\
\hline & 25 & & $4 \mathrm{I} \quad \mathrm{I} 5$ & I I & 34 & & & \\
\hline & 29 & 20 & 5153 & - I I & 35 & o. I $5^{69}$ & $0.233 \mathrm{I}$ & O. I 7 \\
\hline
\end{tabular}

Représentation du lieu moyen $\mathrm{O}-\mathrm{C}$ :

$$
\boldsymbol{\lambda} \boldsymbol{\lambda} \cos \boldsymbol{\beta}=\text { - o. I } \boldsymbol{A} \boldsymbol{\beta}=\text { o.'o. }
$$

On en a déduit l'éphéméride suivante, pour $12^{\mathrm{h}}$ t. m. Paris.

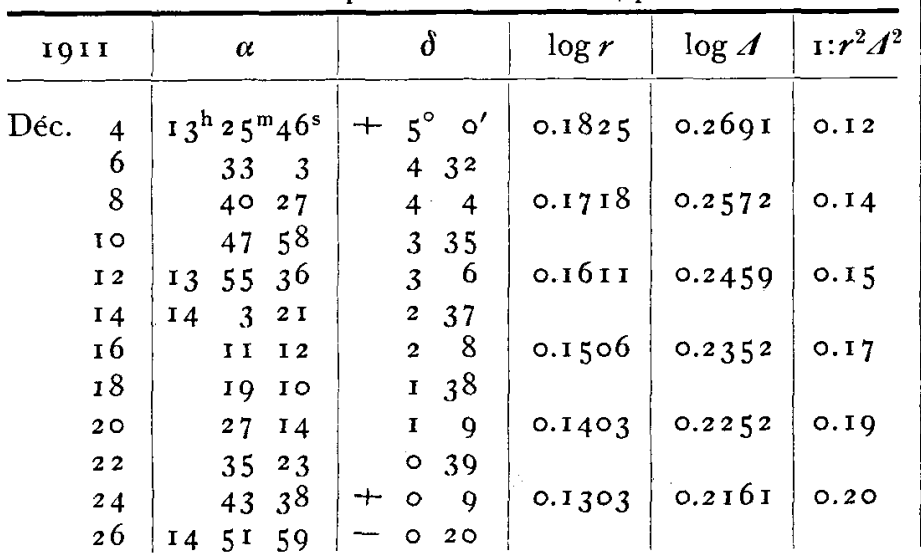

Observatoire de Nice, ı 9 I I Déc. 4 .

G. Fayet. 